\section{Gold(III)-Catalyzed Hydration of Alkynes}

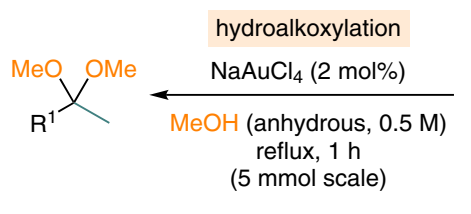

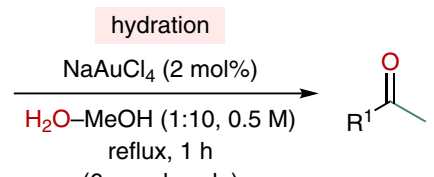

(6 mmol scale)

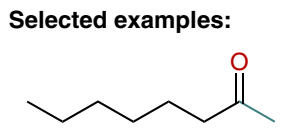

$91 \%$ yield

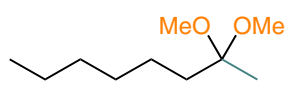

$85 \%$ yield

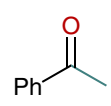

$91 \%$ yield

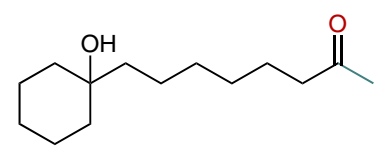

$83 \%$ yield

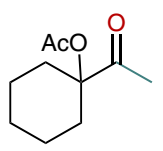

$92 \%$ yield

\section{Key words}

hydration

alkynes

gold catalysis
Significance: In 1991, Fukuda and Utimoto disclosed the gold(III)-catalyzed hydration of alkynes. In contrast to traditional $\mathrm{Hg}(\mathrm{II})$-mediated hydrations that require strongly acidic conditions, the reported $\mathrm{Au}$ (III)-catalyzed protocol is much milder and does not require any acid, allowing for increased functional group tolerance.
Comment: The hydration reaction is operationally simple, requiring only 2 mol\% of $\mathrm{Au}$ (III) catalyst in a refluxing mixture of methanol and water. When strictly anhydrous methanol is used, the corresponding dimethyl acetal product is obtained in place of the ketone.

Review: Z. Li, C. Brouwer, C. He Chem. Rev. 2008, 108, 3239-3265. 